\title{
Encapsulation of Naproxen in Eudragit RSPO Microsphere system: In vitro Characterization and Compatibility Studies
}

\author{
Shovan Paul ${ }^{1}$, Sharmin Akhter ${ }^{2}$, Ikramul Hasan ${ }^{1}$, Syed Shabbir Haider ${ }^{1}$ \\ and Md. Selim Reza \\ ${ }^{1}$ Department of Pharmaceutical Technology, Faculty of Pharmacy, University of Dhaka, Dhaka-1000, \\ Bangladesh. \\ ${ }^{2}$ Department of Pharmacy, University of Asia Pacific, Dhanmondi, Dhaka-1209, Bangladesh
}

\begin{abstract}
The present study has been performed to encapsulate naproxen to provide sustained action and to minimize local side effects by avoiding the drug release in the upper gastrointestinal tract. Naproxen was microencapsulated by oil-in-oil emulsification-solvent evaporation technique using Eudragit RSPO. The formulations were prepared by sequential increase in drug loading ranging from $10 \%$ to $70 \%$. In-vitro drug release was studied in a paddle type dissolution apparatus (USP type II) for six hours in phosphate buffer having $\mathrm{pH}$ 7.4. At $10 \%$ and $70 \%$ loading the release were $42.89 \%$ and $86.33 \%$, respectively. The release mechanisms were explored and explained with zero order, first order, Higuchi and Korsmeyer-Peppas models. The correlation-coefficient values of the trend lines of the graphs showed that the formulations were best fitted with Korsmeyer-Peppas release pattern. Microspheres`surface morphologic study was done by scanning electron microscopy. Drug polymer incompatibility studies were performed by Differential Scanning Calorimetry (DSC) and Fourier Transform Infrared Spectroscopy (FTIR). The absence of endothermic melting peak of naproxen in DSC thermogram revealed that the drug might be dispersed in the polymer as solid solution or in a metastable molecular dispersion. But the chemical integrity of Naproxen was not changed or destroyed within the microsphere which was confirmed by FTIR spectral data.
\end{abstract}

Key words: Eudragit RSPO, Naproxen, Microsphere, Emulsification-Solvent evaporation method.

\section{INTRODUCTION}

Naproxen is a nonsteroidal anti-inflammatory drug (NSAID), mainly used in osteoarthritis, rheumatoid arthritis, and ankylosing spondylitis. Naproxen is rapidly and efficiently absorbed after oral administration but has a short half-life of 1-3 hrs and requires multiple dosing for maintaining therapeutic effect throughout the day. Naproxen extensively bound to plasma albumin, so it is more rational to deliver this drug in sustained-release dosage form. The most frequent adverse side effects occurring with naproxen are gastrointestinal (GI) disturbances, peptic ulceration, and GI bleeding. Therefore, to achieve a more effective therapy, avoiding the large fluctuations in drug concentration and consequent reduction in adverse effects to reduce

Correspondence to: Md. Selim Reza

Tel: +880-2-8612069, Fax: +880-28615583

E-mail: selim.du@gmail.com

Dhaka Univ. J. Pharm. Sci. 11(2): 147-155, 2012 (December) frequency of administrations development of naproxen sustained-release dosage forms is more desirable. ${ }^{1}$

Microspheres are defined as homogeneous, monolithic particles in the size range of about 0.1 $1000 \mu \mathrm{m}$ and are widely used as drug carriers for sustained release. These systems have significant importance in biomedical applications. Microspheres can be produced for protection of core material, reduction of gastric irritation, and decrease in volatility, conversion of liquid to pseudo-solid, cell microencapsulation, and designing of pulsatile drug delivery systems. Administration of the drug in the form of microspheres usually improves the treatment by providing the localization of the active substances at the site of action and by prolonging release of drugs. This technology is mainly used for the purpose of protection, controlled release, and incompatibility of the core materials. ${ }^{2,3}$ 
Microencapsulation is widely used in the pharmaceutical and other sciences to mask tastes or odors, prolong release, impart stability to drug molecules, improve bioavailability, and as multiparticulate dosage forms to produce controlled or targeted drug delivery. It is therefore a rapidly expanding technology for achieving sustained-release dosage forms. The solvent-evaporation method of microencapsulation involves the use of emulsification of a solution containing polymer and drug with an additional medium in which the drug and polymer will not dissolve. The technique was relatively simple and has been used to prepare microspheres of a variety of compounds using several different polymeric materials. The ammonium groups are present as salts and responsible for $\mathrm{pH}$ independent permeability of the polymer. Films prepared from Eudragit RSPO are only slightly permeable to water. ${ }^{4,5}$

Microencapsulation of naproxen was also performed several times previously. Among the different methods of microencapsulation, the most common ones are solvent evaporation, coacervation, interfacial polymerization, spray drying and ionotropic gelation. ${ }^{6}$

\section{MATERIALS AND METHODS}

Naproxen was collected as a gift sample from ACI Pharmaceuticals Limited, Bangladesh. All other ingredients were of analytical grade and collected from local market.

Preparation of naproxen microspheres by emulsification solvent evaporation method using Eudragit-RSPO. At first polymer solution was prepared by using 1:1 ethanol and dichloromethane which acts as internal phase. Then naproxen was suspended in light liquid paraffin oil along with $1 \%$ span-80 using stirrer for 5-10 minutes which acts as external phase. After that, internal phase was poured to external phase drop wise and stirred for $2.5 \mathrm{hrs}$ at $2500 \mathrm{rpm}$. After 2.5 hours, prepared microspheres were washed with cyclohexane repeatedly and allowed to dry at room temperature. Finally the microspheres were transferred into glass vials and kept in the desiccator for further experiment. Here all the factors were kept constant except drug loading which was increased by $10 \%$ in each time up to $70 \%$. The total amount of drug and polymer was fixed to $1500 \mathrm{mg}$. Thus the amount of polymer decreases as the dose increases sequentially.

Table 1. Formulation of naproxen microsphere using EU-RSPO

\begin{tabular}{lccccccc}
\hline Ingredients & F1 & F2 & F3 & F4 & F5 & F6 & F7 \\
\hline Drug (mg) & 150 & 300 & 450 & 600 & 750 & 900 & 1050 \\
EU-RSPO (mg) & 1350 & 1200 & 1050 & 900 & 750 & 600 & 450 \\
Ethanol (ml) & 5 & 5 & 5 & 5 & 5 & 5 & 5 \\
DCM (ml) & 5 & 5 & 5 & 5 & 5 & 5 & 5 \\
Span 80 & $1 \%$ & $1 \%$ & $1 \%$ & $1 \%$ & $1 \%$ & $1 \%$ & $1 \%$ \\
Paraffin (ml) & \multicolumn{7}{c}{ up to $100 \mathrm{ml}$} \\
\hline
\end{tabular}

Determination of drug entrapment efficiency. $50 \mathrm{mg}$ of microspheres was taken in a mortar and was triturated properly until fine powder was formed. 20 $\mathrm{mg}$ fine powder was taken in a screw cap test tube. 5 $\mathrm{ml}$ buffer was added to the powdered microsphere and was vortexed for 10 minutes. Then the solution was filtered and taken in a $100 \mathrm{ml}$ volumetric flask. The volume of the solution adjusted to $100 \mathrm{ml}$ with the buffer solution. The absorbance was taken at 271 $\mathrm{nm}$. From the absorbance value the amount of naproxen entrapped was determined using standard curve. The drug entrapment efficiency was calculated by using the following equation-

$$
\text { Entrapment efficiency }(\%)=\frac{\text { Calculated drug concentration }}{\text { Theoretical drug concentration }} \times 100
$$

In vitro dissolution study of Eudragit-RSPO microspheres containing naproxen. In-vitro dissolution study was performed in a paddle type (Type II) dissolution apparatus. $100 \mathrm{mg}$ of microsphere was taken from each batch of formulation for dissolution study. Phosphate buffer of $\mathrm{pH} 7.4$ as mentioned in BP was used as dissolution media, paddle speed was set at $50 \mathrm{rpm}$ and temperature was maintained at $37{ }^{\circ} \mathrm{C}$. The fixed amount of microspheres from each batch was weighed and transfered in each dissolution basket. The dissolution process was carried out for 6 hours and $10 \mathrm{ml}$ dissolution sample from each dissolution 
media was withdrawn at a predetermined intervals of 15 minutes, 30 minutes, 1 hour, 2 hours, 3 hours, 4 hours, 5 hours and 6 hours. Each and everytime 10 $\mathrm{ml}$ of dissolution sample was compensated by fresh $10 \mathrm{ml}$ phosphate buffer ( $\mathrm{pH}$ 7.4). Dissolution samples were withdrawn and then filtered through $0.45 \mu \mathrm{m}$ sized disposable syringe. The dissolution samples were then analyzed spectrophotometrically at $271 \mathrm{~nm}$ in a UV-VIS spectrophotometer.

Surface morphologic study by scanning electron microscope (SEM). Scanning electron microscopic study has been employed to study the morphology and surface topography of the microspheres. The microspheres were mounted on the SEM sample stab (aluminium stabs) which were coated with a double-sided sticking tape, sealed and finally coated with gold $(200 \AA)$ under reduced pressure (0.001 torr) for 15 minutes using an ion sputtering device. The gold coated samples were scanned using scanning electron microscope (S$3400 \mathrm{~N}$, Hitachi) under varying magnifications and photomicrographs of suitable magnifications were obtained. The microspheres were dried completely before examination.

Fourier transform infrared spectroscopy (FTIR). The IR spectra of the pure drug and microspheres were obtained to prove the chemical integrity of the drug in the microspheres. The samples (about $5 \mathrm{mg}$ ) were powdered and intimately mixed with $250 \mathrm{mg}$ of pure dry powdered potassium bromide and the mixture was pressed into a disc using a special mould and a hydraulic press. The resultant mixtures were taken in a diffuse reflectance sampler and IR spectra recorded by scanning in the wavelength region of 100 to $1000 \mathrm{~cm}^{-1}$ in a FTIR Spectrophotometer (model 460 Plus, Jasco, Japan).

Thermal analysis by differential scanning calorimetry (DSC). Differential scanning calorimetry (DSC) is a thermo-analytical technique in which the difference in the amount of heat is required to increase the temperature of a sample and a reference is measured as a function of time. Both the sample and reference are maintained at nearly the same temperature throughout the experiment.
Generally the temperature programmed for DSC analysis is designed such that the sample holder temperature increases linearly as a function of time. The reference sample should have a well defined heat capacity over the range of temperatures to be scanned.

\section{RESULTS AND DISCUSSION}

\section{Drug loading and drug incorporation} efficiency. Eudragit RSPO has excellent incorporation efficiency as all the formulations showed incorporation efficiency above $97 \%$.

\begin{tabular}{cc}
\hline Formulations & $\begin{array}{c}\text { Entrapment } \\
\text { Efficiency }(\%)\end{array}$ \\
\hline F1 & 99.3 \\
F2 & 98.41 \\
F3 & 97.94 \\
F4 & 99.25 \\
F5 & 98.67 \\
F6 & 99.8 \\
F7 & 99.31 \\
\hline
\end{tabular}

Effect of Eudragit-RSPO on release kinetics of naproxen from microspheres. For finding the release profile dissolution testing of the EudrgitRSPO microspheres containing naproxen of all formulations (F1 to F7) were carried out in phosphate buffer ( $\mathrm{pH}$ 7.4) for 6 hours at $50 \mathrm{rpm}$ and release rate of naproxen in phosphate buffer was calculated. The release kinetics has been shown in figure 1. Different release profile were plotted to find out the best fitted models and to observe the release pattern of naproxen from the Eudragit-RSPO microspheres.

From the zero order release kinetics, it can be observed that the release rate of naproxen were $42.89,49.15,53.74,58.01,66.76,76.43$ and $86.33 \%$ within 6 hours for F1, F2, F3, F4, F5, F6 and F7, respectively. Figure 1B shows that decreasing the percentage of polymer from 90 to $30 \%$ resulted in a marked increase in drug release rate.

Release profiles of naproxen from EudragitRSPO microspheres considering $\mathbf{R}^{2}, \mathbf{n}, \mathbf{T}_{25 \%}, \mathbf{T}_{50 \%}$, $\mathbf{T}_{\mathbf{8 0} \%}$ and MDT values. From table 2 it is observed that all the formulations (F1 to F7) was best fitted to the Korsmeye-Peppas model. The values of release exponent (n) of the formulations were below 0.45 and 
the value of $n$ reduced with the decrease of concentration of polymer. These values indicates that the drug was released following Fickian release pattern, which means the zero order was changed over time. $^{7}$
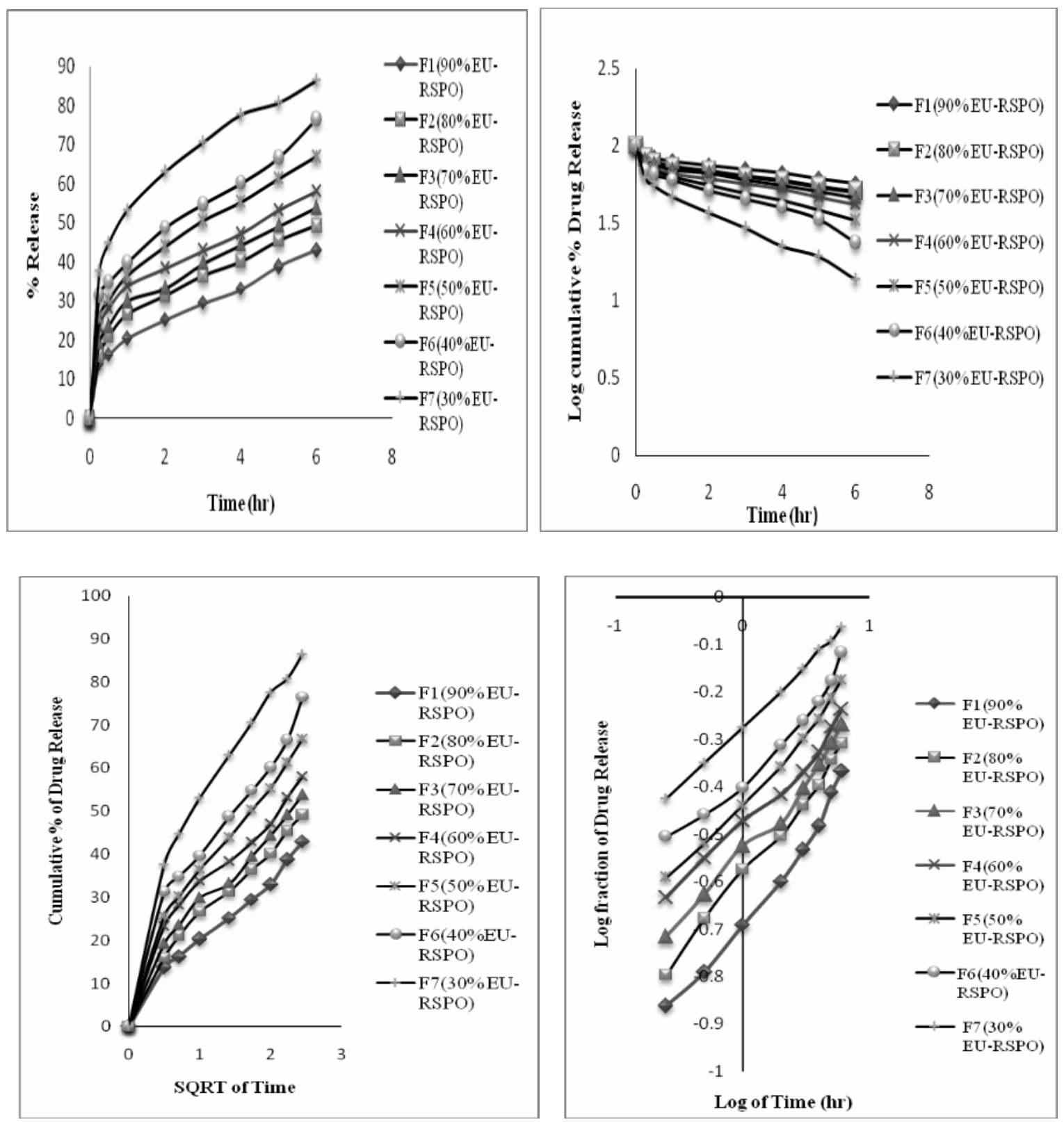

Figure 1. In vitro release kinetics of naproxen from Eudragit-RSPO microspheres: A. Zero order plot, B. First order plot, C. Higuchi plot, D. Korsmeyer plot

As the microspheres come in contact with the phosphate media there is burst release of surface drug forming pores on the surface. Subsequently, the media enters through pores and initiate Fickian diffusion of the rest of the drug whose release rate changes with time. The successive fractional dissolution time values were shown in table 3. It can be observed from the values that $\mathrm{T}_{25 \%}, \mathrm{~T}_{50 \%}, \mathrm{~T}_{80 \%}$ and 
MDT of F1 to F7 gradually decrease. As the concentration of polymer decreases the drug release rate gradually increases and the values of $\mathrm{T}_{25 \%}, \mathrm{~T}_{50 \%}$, $\mathrm{T}_{80 \%}$ and MDT gradually becomes lower.

Eudragit RSPO polymer is a series of acrylate and methacrylate polymers available in different ionic forms. Eudragit RSPO polymer belongs to the class of poly methacrylates polymers that are insoluble but permeable in digestive fluids. They possess alkaline functional groups which lend themselves to $\mathrm{pH}$ independent swelling (in the physiological range) and enable sustained release of active ingredient in the formulation. ${ }^{8,9}$

Table 2. Interpretation of release rate constants and $\mathbf{R}^{2}$ values for different release kinetics of Eudragit-RSPO based naproxen micro spheres.

\begin{tabular}{cccccccccc}
\hline \multirow{2}{*}{ Formulations } & \multicolumn{2}{c}{ Zero order } & \multicolumn{2}{c}{ First order } & \multicolumn{2}{c}{ Higuchi } \\
\cline { 2 - 7 } & $\mathrm{K}_{0}$ & $\mathrm{R}^{2}$ & $\mathrm{~K}_{1}$ & $\mathrm{R}^{2}$ & $\mathrm{~K}_{\mathrm{H}}$ & $\mathrm{R}^{2}$ & $\mathrm{n}$ & 0.97 & 0.351 \\
\hline F1 & 5.754 & 0.892 & -0.033 & 0.936 & 15.8 & 0.98 \\
F2 & 6.515 & 0.849 & -0.04 & 0.914 & 18.26 & 0.973 & 0.337 & 0.993 \\
F3 & 6.595 & 0.839 & -0.45 & 0.915 & 19.53 & 0.964 & 0.311 & 0.985 \\
F4 & 7.139 & 0.796 & -0.049 & 0.892 & 20.3 & 0.939 & 0.27 & 0.983 \\
F5 & 8.538 & 0.824 & -0.065 & 0.93 & 24.12 & 0.959 & 0.295 & 0.99 \\
F6 & 9.342 & 0.818 & -0.081 & 0.931 & 26.28 & 0.944 & 0.271 & 0.964 \\
F7 & 10.66 & 0.752 & -0.121 & 0.946 & 30.28 & 0.925 & 0.26 & 0.998 \\
\hline
\end{tabular}

Table 3. Successive fractional dissolution times (hours) of F1 to F7.

\begin{tabular}{ccccc}
\hline \multirow{2}{*}{ Formulations } & \multicolumn{4}{c}{ Successive dissolution time (hours) } \\
\cline { 2 - 4 } & $\mathrm{T}_{25 \%}$ & $\mathrm{~T}_{50 \%}$ & MDT & $\mathrm{T}_{80 \%}$ \\
\hline F1 & 1.65 & 11.86 & 22.20 & 45.24 \\
F2 & 0.90 & 7.06 & 13.91 & 28.46 \\
F3 & 0.61 & 5.70 & 12.55 & 25.81 \\
F4 & 0.34 & 4.41 & 12.21 & 25.14 \\
F5 & 0.26 & 2.69 & 6.43 & 13.25 \\
F6 & 0.14 & 1.84 & 5.07 & 10.44 \\
F7 & 0.05 & 0.77 & 2.29 & 4.71 \\
\hline
\end{tabular}

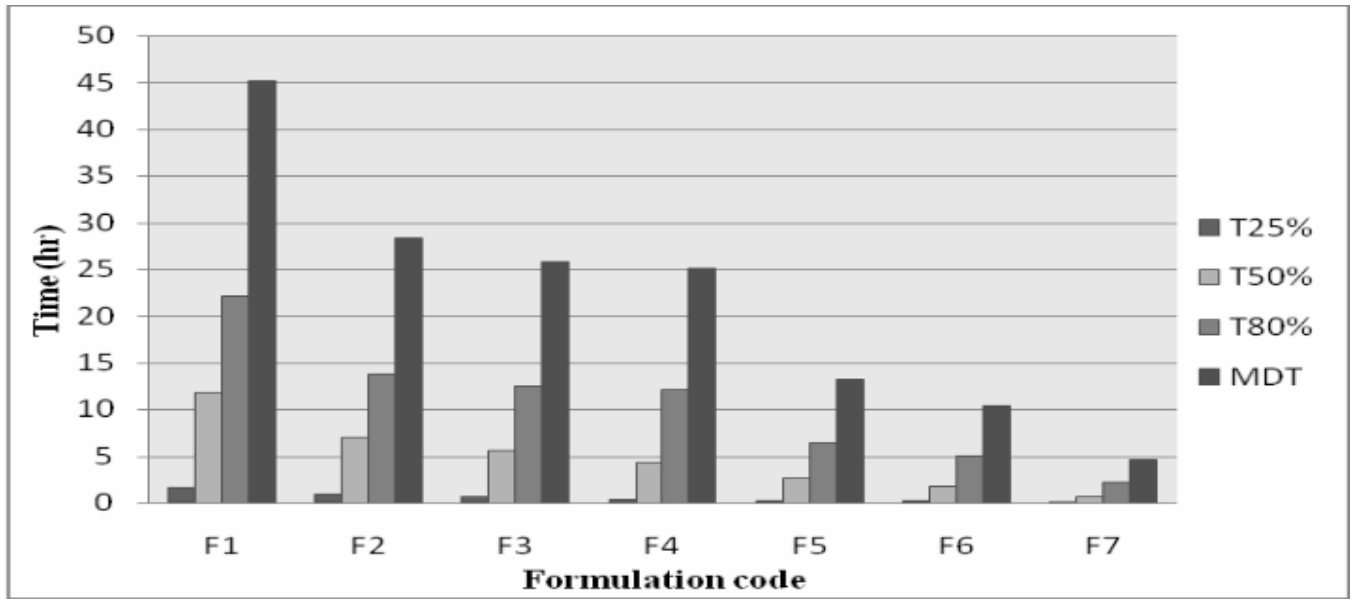

Figure 2. Bar diagram of successive fractional dissolution time.

Effect of Eudragit-RSPO on the surface morphology of F1 microsphere. Microspheres of Eudragit containing naproxen were prepared by using emulsification solvent evaporation technique. F1 was observed by SEM (SEM, S-3400N, Hitachi) to see the morphological changes that occurred due to formulation variation. A SEM image of F1 was taken and surface of the microspheres were analyzed. The image showed uneven surface of microspheres of $\mathrm{F} 1$ (shown in figure $3 \mathrm{~A}$ ). Figure $3 \mathrm{C}$ showed small 
amount of surface drug and slight pores on the surface. This was due to very small amount of drug loading (which was only $10 \%$ in case of F1).
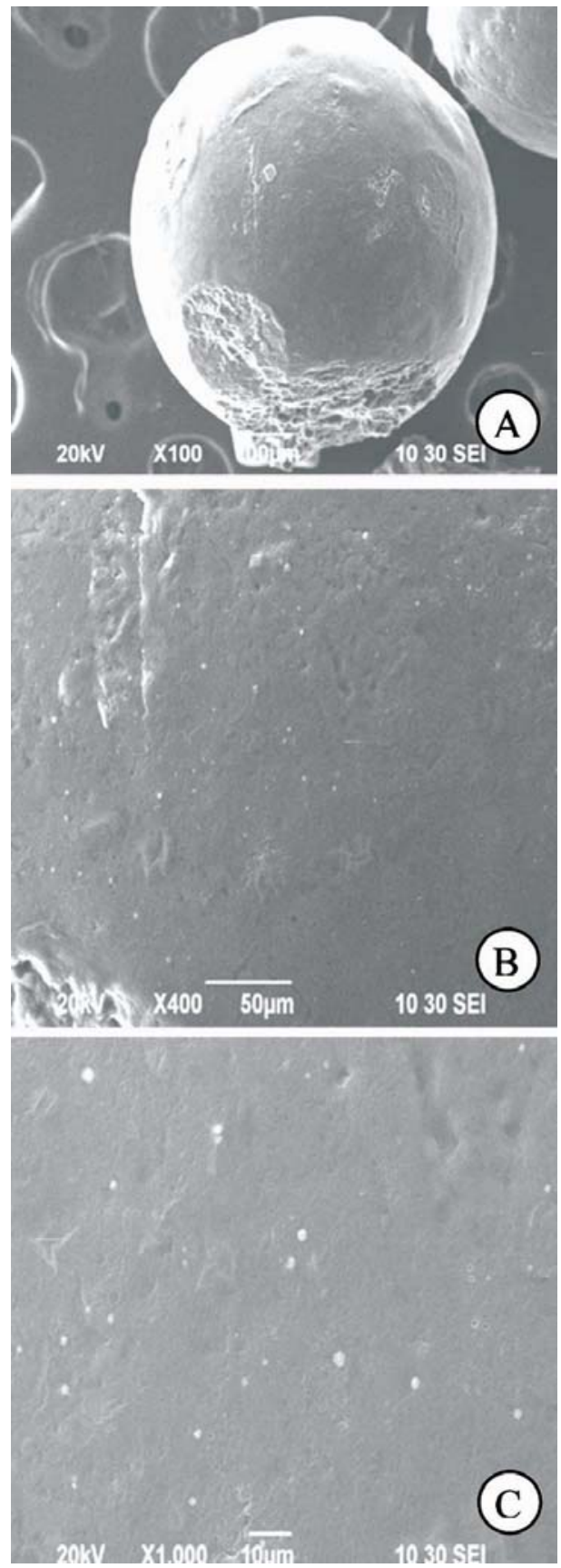

Figure 3. Effect of Eudragit-RSPO on surface Morphology of F1 micro spheres: A. Magnification at X100 SE; B. Magnification at X400 SE and C. Magnification at X1000SE.

\section{Compatibility Study}

Fourier transform infrared spectrometric (FTIR) study to determine compatibilit.

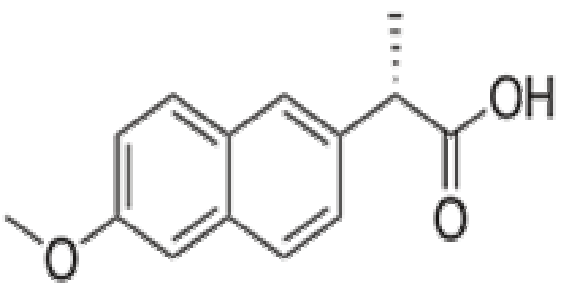

Figure 4. Chemical structure of Naproxen.

FTIR spectrum of pure Naproxen. According to the chemical structure of Naproxen shown in figure 4 it contains aromatic ring, carboxylic group, alkane group and methoxy group.

\begin{tabular}{|c|c|c|c|c|}
\hline $\begin{array}{l}\text { Functional } \\
\text { gorup }\end{array}$ & & $\begin{array}{l}\text { Standard } \\
\text { peak } \\
\text { eegion }\end{array}$ & $\begin{array}{l}\text { FTIR spectra } \\
\text { of pure } \\
\text { naproxen }\end{array}$ & $\begin{array}{l}\text { FTIR } \\
\text { spectra } \\
\text { of F7 }\end{array}$ \\
\hline \multirow{4}{*}{$\begin{array}{l}\text { Carboxyl } \\
\text { Group } \\
(-\mathrm{COOH})\end{array}$} & $\mathrm{O}-\mathrm{H}$ & $3500-$ & $3214 \mathrm{~cm}^{-1}$ & 3443.96 \\
\hline & Stretch & $2400 \mathrm{~cm}^{-1}$ & & $\mathrm{~cm}^{-1}$ \\
\hline & $\begin{array}{l}\mathrm{C}=\mathrm{O} \\
\text { stertch }\end{array}$ & $\begin{array}{l}1730- \\
1700 \mathrm{~cm}^{-1}\end{array}$ & $1227.71 \mathrm{~cm}^{-1}$ & $\begin{array}{l}1729.21 \\
\mathrm{~cm}^{-1}\end{array}$ \\
\hline & $\begin{array}{l}\mathrm{C}-\mathrm{O} \\
\text { stretch }\end{array}$ & $\begin{array}{l}1320- \\
1210 \mathrm{~cm}^{-1}\end{array}$ & $\begin{array}{l}1729.210 \\
\mathrm{~cm}^{-1}\end{array}$ & $\begin{array}{l}1304.91 \\
\mathrm{~cm}^{-1}\end{array}$ \\
\hline Aromatic & $\mathrm{C}=\mathrm{C}-\mathrm{C}^{\mathrm{a}}$ & $1615-$ & $1604.8 \mathrm{~cm}^{-1}$ & 1604.80 \\
\hline Ring & stretch & $\begin{array}{l}1580 \mathrm{~cm}^{-1} \\
\text { and } \\
1510-1450 \\
\mathrm{~cm}^{-1} .\end{array}$ & $\begin{array}{l}1510-1450 \\
\mathrm{~cm}^{-1}\end{array}$ & $\begin{array}{l}\mathrm{cm}^{-1} \\
\text { and } \\
1453.39 \\
\mathrm{~cm}^{-1}\end{array}$ \\
\hline \multirow[t]{2}{*}{ Ether } & $\begin{array}{l}\text { Aryl-O } \\
\text { stretch }\end{array}$ & $\begin{array}{l}1270- \\
1230 \mathrm{~cm}^{-1}\end{array}$ & $1264.36 \mathrm{~cm}^{-1}$ & $\begin{array}{l}1264.36 \\
\mathrm{~cm}^{-1}\end{array}$ \\
\hline & $\begin{array}{l}\text { Alkyl } \\
\text { C-O } \\
\text { stretch }\end{array}$ & $\begin{array}{l}1150- \\
1050 \mathrm{~cm}^{-1}\end{array}$ & $1090.76 \mathrm{~cm}^{-}$ & $\begin{array}{l}1029.04 \\
\mathrm{~cm}^{-1}\end{array}$ \\
\hline
\end{tabular}

The peak for the prominant functional groups of naproxen were also found in the FTIR spectra of naproxen microsphere indicated successful entrapment of naproxen within the micro spheres.

Differential scanning calorimetric (DSC) study to determine compatibility. Thermal analysis of Naproxen, Eudragit RSPO, Physical mixture of Eudragit RSPO and Naproxen and Naproxen loaded Eudragit RSPO Microsphere:

Figure 6 shows the thermal behavior of pure Naproxen (Figure 6A), pure Eudragit RSPO (Figure 6B), physical mixture of Eudragit RSPO and Naproxen (Figure 6C) and Naproxen loaded Eudragit RSPO microspheres (Figure 6D). Pure naproxen showed an endothermic melting peak at $157.35^{\circ} \mathrm{C}$. Pure polymer (Eudragit RSPO) showed an endothermic melting peak at $103.39{ }^{\circ} \mathrm{C}$. In the 
physical mixture endothermic peaks of Eudragit and naproxen were found. But naproxen's endothermic melting peak disappeared in naproxen loaded Eudragit microsphere (Figure 6D), indicating the absence of drug's crystalline state in the delivery system. During microsphere formation, the polymer inhibited the recrystallisation of the drug. ${ }^{10}$
According to Dubernet the disappearance of the endothermic peak, correspondent to the encapsulated drug melting point, indicates the drug is dispersed in the polymer as solid solution or that the drug is dispersed in the polymer in a metastable molecular dispersion.
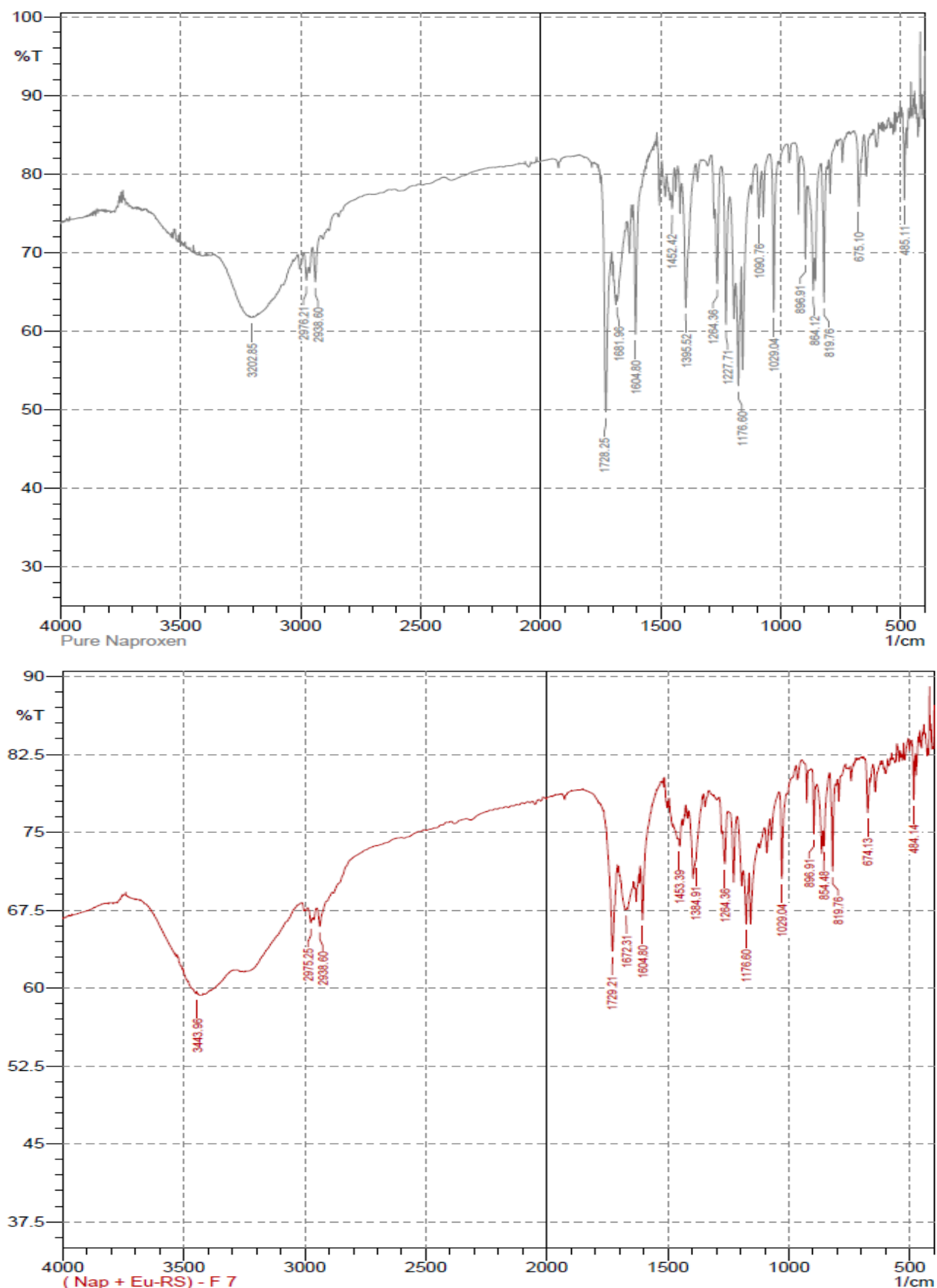

Figure 5. FTIR spectrum of A. pure Naproxen; B. F7 microspheres (70\% loading, 30\% Eudragit-RSPO). 


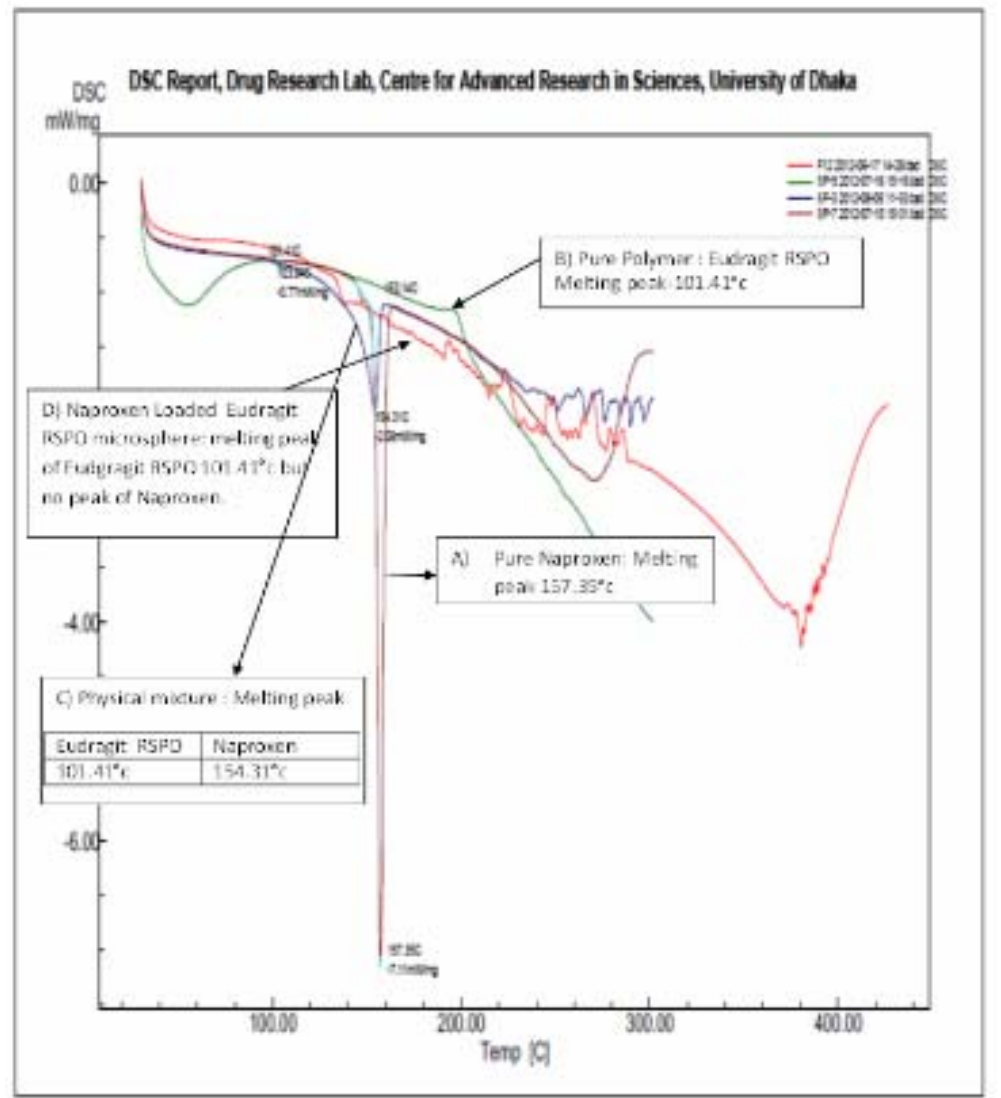

Figure 6. Thermal analysis of Naproxen, Eudragit RSPO, physical mixture of Eudragit RSPO and Naproxen and Naproxen loaded Eudragit RSPO microsphere.

\section{CONCLUSION}

Eudragit-RSPO can be an excellent candidate for encapsulating naproxen which has successfully been established throughout this research work. Microspheres were prepared by emulsificationsolvent evaporation technique which was found to be reproducible and also may be an ideal method to prepare microspheres in large sale. The results of dissolution study showed all the formulations were best fitted to Korsmeyer-Peppas model and follow Fickian release pattern. The SEM reports depict that the particle surface morphology changes with the change of drug loading. A high loading results in a high surface drug. The DSC indicates the drug is dispersed in the polymer as solid solution or that the drug is dispersed in the polymer in a metastable molecular dispersion. The FTIR revealed the compatibility between the drug and the polymer.

\section{REFERENCES}

1. Zaghloul, A.A., Faltinek, J., Vaithiyalingam, S.R., Reddy, I.K. and Khan, M.A. 2001. Screening of process and formulation variables for the preparation of extended release tablets of Naproxen-Eudragit microspheres. Pharmazie. 56, 321-4.

2. Wan, LS. and Chui, WK. 1995. Deviation of the ratio of drugs in a two component mixture encapsulated in cellulose phthalate microspheres. J. Microencapsul. 12, 417-23.

3. Yamuda, T., Onishi, H. and Machida, Y. 2001. Sustained release ketoprofenmicroparticles with ethyl cellulose and carboxymethyl ethyl cellulose. J. Controlled Release. 75, 271-282.

4. Bolourtchian, N., Karimi, K. and Aboofazeli, R. 2005. Preparation and characterization of ibuprofen microspheres. J. Microencapsul. 22, 529-538.

5. Haznedar, S., Dortunc, B. 2004. Preparation and in vitro evaluation of Eudragit microspheres containing acetazolamide. Int. J. Pharm. 269, 131-140. 
6. Horoz, B., Kiliic, M., Arslan, N., Baykara, Y T. 2004. Effect of different dispersing agents on the characteristics of Eudragit microspheres prepared by a solvent evaporation method. J. Microencapsul. 21, 191-202.

7. Haznedar, S.,and Dortue, B. 2004. Preparation and in vitro evaluation of Eudragit microspheres containing acetazolamide. Intl. J. Pharm. 269, 131-140.

8. Higuchi, T. 1963. Mechanism of sustained action medication, theoretical analysis of rate of release of solid drugs dispersed in solid matrices. J. Pharm. Sci. 52, 1145-1149.
9. Korsmeyer, RW., Gurny, R., Doelker, EM., Buri, P. and Peppas, N.A. 1983. Mechanism of solute release from porous hydrophilic polymers. Intl. J. Pharm. 15, 25-35.

10. Mello, V.A. and Ricci, R. 2011. Encapsulation of Naproxen in nanosphered system: structural characterization and in vitro release studies Vol. 34, no. 6 . 